Article

\title{
Improvement of Polytetrafluoroethylene Membrane High-Efficiency Particulate Air Filter Performance with Melt-Blown Media
}

\author{
Euijin Shim ${ }^{1}$, Jeong-Phil Jang ${ }^{2,3}$, Jai-Joung Moon ${ }^{3}$ and Yeonsang Kim ${ }^{1, *}$ \\ 1 Advanced Textile R\&D Department, Korea Institute of Industrial Technology (KITECH), \\ 143 Hanggaulro, Sangnok-gu, Ansan-si 15588, Korea; pelice35@kitech.re.kr \\ 2 Department of Advanced Organic Materials Engineering, Jeonbuk National University, \\ 567 Baekje-daero, Deokjin-gu, Jeonju-si 54896, Korea; jpjang@cands.kr \\ 3 Clean and Science Co., Ltd., 67, 3sandan 3-gil, Buk-myeon, Jeongeup-si 56137, Korea; jjmoon@cands.kr \\ * Correspondence: yskim@kitech.re.kr
}

check for updates

Citation: Shim, E.; Jang, J.-P.; Moon, J.-J.; Kim, Y. Improvement of Polytetrafluoroethylene Membrane High-Efficiency Particulate Air Filter Performance with Melt-Blown Media. Polymers 2021, 13, 4067. https:// doi.org/10.3390/polym13234067

Academic Editor: Dagmar R. D’hooge

Received: 6 October 2021

Accepted: 22 November 2021

Published: 23 November 2021

Publisher's Note: MDPI stays neutra with regard to jurisdictional claims in published maps and institutional affiliations.

Copyright: (c) 2021 by the authors. Licensee MDPI, Basel, Switzerland. This article is an open access article distributed under the terms and conditions of the Creative Commons Attribution (CC BY) license (https:// creativecommons.org/licenses/by/ $4.0 /)$

\begin{abstract}
Polytetrafluoroethylene (PTFE) membrane filters are widely used in low-load application areas, such as industrial cleanrooms, due to their low initial pressure drop. In this study, meltblown (MB) nonwoven was introduced as a pre-filtration layer at the front end of a high-efficiency particulate air (HEPA) filter to improve the filter performance of the PTFE membrane. Pre-filtration reduces the average particle size, which reaches the PTFE membrane and reduces the dust load on the HEPA filters. A comparative analysis of the HEPA filters by composite MB and PTFE was conducted. Regarding the MB composite on the PTFE, low-weight and high-weight MB composites were effective in increasing dust filtration efficiency, and the dust loading capacity of the PTFE composite with high-weight MB increased by approximately three times that of the PTFE membrane. In addition, the filter was installed on an external air conditioner in an actual use environment and showed a high efficiency of $99.984 \%$ without a change in differential pressure after 120 days.
\end{abstract}

Keywords: HEPA filter; melt-blown; pre-filter; PTFE membrane; clean room

\section{Introduction}

High-efficiency particulate air (HEPA) filters have been used in various fields to maintain a clean air environment for industrial facilities, such as producing semiconductor and LCD panels, bio-pharmacies, and nuclear power plants [1-3]. In general, glass fibre has been applied to HEPA filter media. However, polytetrafluoroethylene (PTFE) membranes used to remove particles in industries have recently been developed to have the same efficiency level as glass fibre. PTFE has emerged as an alternative option with a much lower initial pressure drop [3,4].

PTFE membranes exhibit excellent chemical resistance, thermal stability, and strong hydrophobicity owing to the strong $\mathrm{C}-\mathrm{C}$ and $\mathrm{C}-\mathrm{F}$ bonds. The combination of strong bonds, a protective sheath, and non-polarity not only makes PTFE thermally stable but also inactive. These features are suitable for a variety of applications, such as fine dust filtration, exhaust gas treatment, and membrane distillation. PTFE, a microporous membrane, is a valuable air filter medium with a high filtration efficiency and flow velocity $[5,6]$. The porous membrane filter material refers to many small pores in the membrane matrix, such as the expanded PTFE (ePTFE) membrane. Owing to its low porosity, this type of material has low airflow and generally has an exceedingly high filtration effect but extremely high air resistance $[7,8]$.

The HEPA filter determines the filtration performance by the HEPA filter media, which is characterised by two parameters: filtration efficiency and pressure drop. These parameters increase as dust accumulates in the filter medium. Long-term pressure drop performance is important, especially for filter performance, as increased pressure drop 
affects increased energy consumption and determines filter life [3]. Thus, the dust loading capacity is a particularly important parameter in filter design. It is necessary to consider the particle loading characteristics at the filter design stage $[9,10]$.

Previous researchers developed a three-layer filter in which each layer showed a different fibre density to achieve high collection efficiency and low pressure drop and reported the filtration performance dust holding capacity of the filter [10,11].

Although there are studies on layering melt-blown nonwoven (hereafter MBNW) as a pre-filter [12,13], no study has applied MB as a pre-filter to PTFE membranes to demonstrate their high filter characteristics. Thus, this study compared the pre-filter effect of the HEPA filter produced by filtering the PTFE membrane under MB production conditions. The pre-filter reduces the average particle size that reaches the PTFE membrane while reducing the dust load on the HEPA filter [14,15]. The basic properties, such as thickness, weight, and air permeability, of the filter media were analysed. This study quantified the consequential effects of the overall pressure drop and filter lifespan. Finally, the industrial application was confirmed through demonstration experiments in a real usage environment.

\section{Materials and Methods}

\subsection{Materials}

MBNW media samples were produced from commercial grade polypropylene (PP). PP resin (melt index: $1200 \mathrm{~g} / 10 \mathrm{~min}$ ) was purchased from LG Chem Co., Ltd. (Seoul, Korea). A melt blowing pilot line from Clean and Science Co was used, and the PTFE membrane was produced by Micro-One Co. (Cheonan, Korea) The average thickness of the PTFE membrane was $4.33 \mu \mathrm{m}$. Low-melting polyethylene terephthalate (PET) and a nonwoven PE/PET blend were used as the PTFE support.

\subsection{Preparation of the High-Efficiency Particulate Air Filter with Melt-Blown Nonwoven}

MBNW is a major process used to manufacture fibrous nonwoven fabrics. It was used as a pre-filter to prevent nanoparticles from passing through the PTFE membrane layer. The PP-based MBNW fabric was prepared using a pilot-scale melt-injection setup (Figure 1). The process temperature of the extruder was controlled using a two-component nozzle to $200-250^{\circ} \mathrm{C}$. The MB-grade PP pellets were placed into a twin-screw extruder, and the output was controlled by the speed of the gear pump. The melted polymer was sprayed in the form of single fibres and solidified from the collector. In addition, the net was electrically charged on the MB side by a corona discharger to further improve the filtration efficiency. Four types of MBNW media, as detailed in Table 1, were tested in this study. Finally, the HEPA filter media were fabricated with the MB pre-filter layer and PTFE membrane layer.

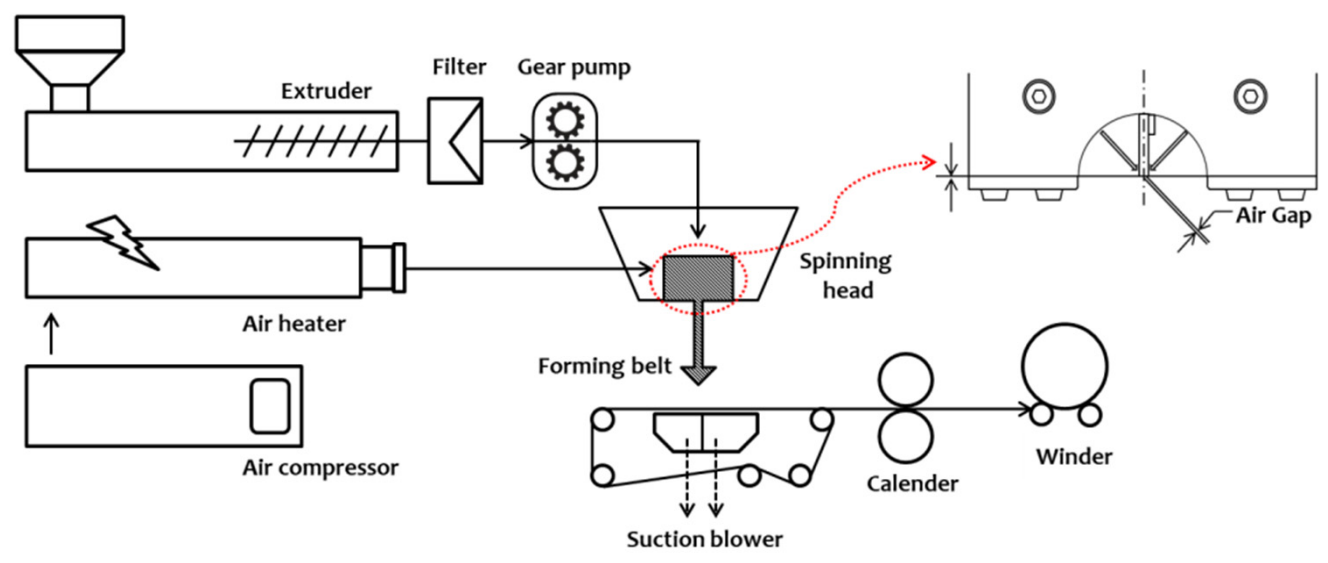

Figure 1. Schematic of a melt-blown spinning process. 
Table 1. Characteristics of tested melt-blown media.

\begin{tabular}{cccc}
\hline Sample & Weight $(\mathbf{g s m})$ & Thickness $(\boldsymbol{\mu m})$ & Fibre Diameter $(\mu \mathrm{m})$ \\
\hline $\mathrm{MB}_{1}$ & 10 & $55 \pm 3$ & $1.80 \pm 0.42$ \\
$\mathrm{MB}_{2}$ & 15 & $79 \pm 2$ & $1.61 \pm 0.29$ \\
$\mathrm{MB}_{3}$ & 20 & $99 \pm 6$ & $1.92 \pm 0.29$ \\
$\mathrm{MB}_{4}$ & 22 & $110 \pm 2$ & $1.97 \pm 0.33$ \\
\hline gsm: gram per square meter. & &
\end{tabular}

The manufactured MBNW and PTFE membranes were laminated to form composites. The HEPA filter unit to be installed in a commercial heating, ventilation, and air conditioning (HVAC) system was fabricated from a PTFE/MB composite pleated and assembled into an aluminium frame.

\subsection{Characterization}

The physical properties of the PTFE membrane and MB composite, such as thickness, pore size, and air permeability, were evaluated. Their thickness was determined using a thickness tester (No.20465, Mitutoyo Co., Kawasaki, Japan) according to the ASTM D 5729-9 standard. The pore sizes distribution of these specimens were measured using a capillary flow porometer (CFP-1500-AEX, PMI Inc., Ithaca, NY, USA) according to the ASTM F316-03 standard. Air permeability was measured using an air permeability tester (FX3300, TexTest Instruments, Zürich, Switzerland) at a constant pressure drop of $125 \mathrm{~Pa}$. Surface images of the PTFE membrane and MB were observed by field emission scanning electron microscopy (FE-SEM; SU8010, Hitachi Co., Tokyo, Japan) with an acceleration voltage of $10 \mathrm{kV}$ after sputter coating with osmium (Os). The static water contact angle (WCA) of the filter media was determined following the sessile drop method using a contact angle analyzer (DSA100, Krüss Co., Hamburg, Germany).

\subsection{Filtration Test}

The efficiencies of the HEPA filter were evaluated using different types of particles and measuring instruments.

First, the filtration efficiency and resistance of the PTFE and PTFE/MB composite filter media were measured using an automated particulate filtration tester (TSI Inc., Shoreview, MN, USA). Poly ( $\alpha$-olefin) (PAO) was selected as the experimental particle and represented oily aerosol dust. The tests were based on the EN 1822 Part 3 standards. The filtration efficiency $(E)$ was calculated based on the aerosol concentration upstream $\left(C_{u p}\right)$ and the aerosol concentration downstream $\left(C_{\text {down }}\right)$ as follows [16,17]:

$$
E=\left(C_{u p}-C_{\text {down }}\right) / C_{\text {up }}
$$

Second, for PTFE membrane and PTFE/MB composite media, the dust loading pattern at $2 \mathrm{wt.} \%$ concentration of $\mathrm{NaCl}$ aerosol and $5.33(\mathrm{~cm} / \mathrm{s})$ face velocity was determined to investigate the effect of the MB pre-filtration layer and MB weight on the HEPA filter loading process. An experiment on the dust load performance comparison experiment between PTFE/MB composite media and PTFE only media was conducted, where $\mathrm{NaCl}$ solid particles were loaded for $60 \mathrm{~min}$ under the same operating conditions.

Third, HVAC environment tests were conducted in an external air conditioner $(1680 \mathrm{cmm})$ environment. The wind speed was $56 \mathrm{cmm}$, and after the initial differential pressure and efficiency were measured, their changes were observed at regular intervals. The tests were measured according to the EN 1822 Part 1 standards. The efficiency was calculated based on the Equation (1) for $0.3 \mu \mathrm{m}$ particles. In this study, the results were measured and presented after 120 days [18]. 


\section{Results and Discussion}

\subsection{Effect of Melt-Blown Physical Properties for High-Efficiency Particulate Air Filter}

In this study, the physical properties according to weight were compared and analysed before MBNW was introduced into the PTFE filter media. This is due to the fact that the MB structure, porosity, and air permeability are factors that can affect filter performance, that is, differential pressure and efficiency $[13,19]$.

\subsubsection{Morphology Structure}

SEM micrographs were obtained at a magnification of 1000 (Figure 2). The bonds of the fibres can be observed in the SEM images. It can also be observed that the diameters of the fibres formed in the process are diverse, as observed in the typical MB webs of other polymers. The fibre orientation was random depending on the processing conditions used. Fibre tangles are successfully obtained at high levels, which affect the pore size required for filtration applications and other controlled barrier fabrics [19]. In addition, it was confirmed that the higher the weight, the higher the density observed with the naked eye.

$\mathrm{MB}_{1}$

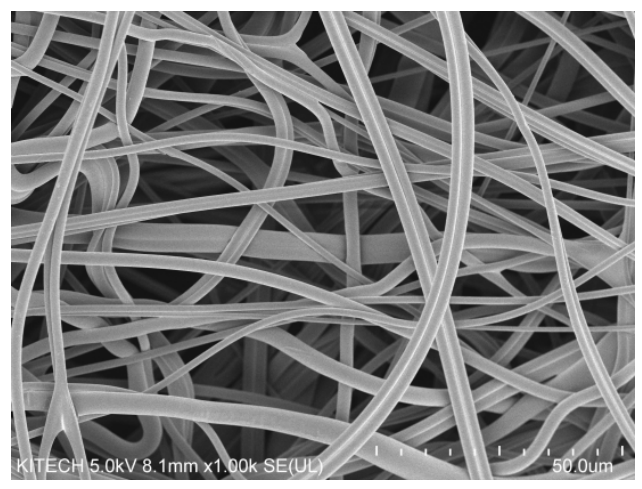

$\mathrm{MB}_{3}$

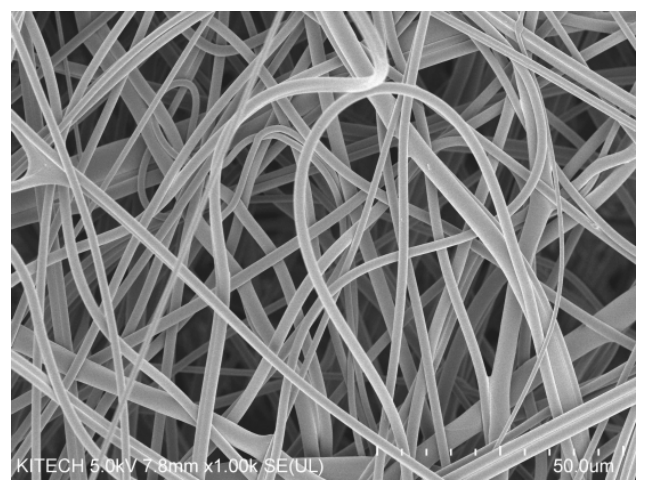

$\mathrm{MB}_{2}$

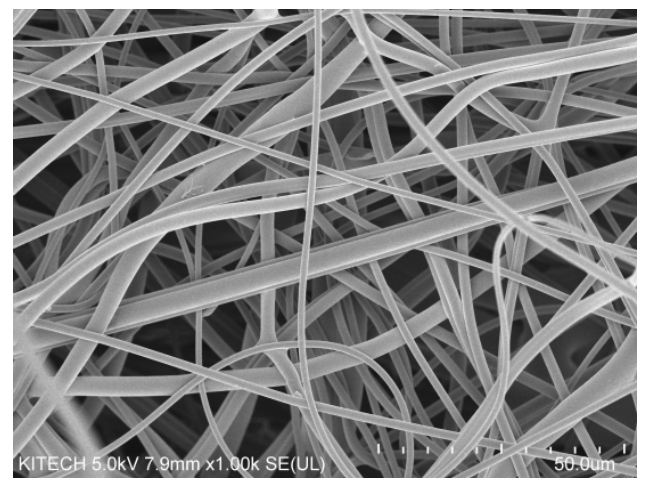

$\mathrm{MB}_{4}$

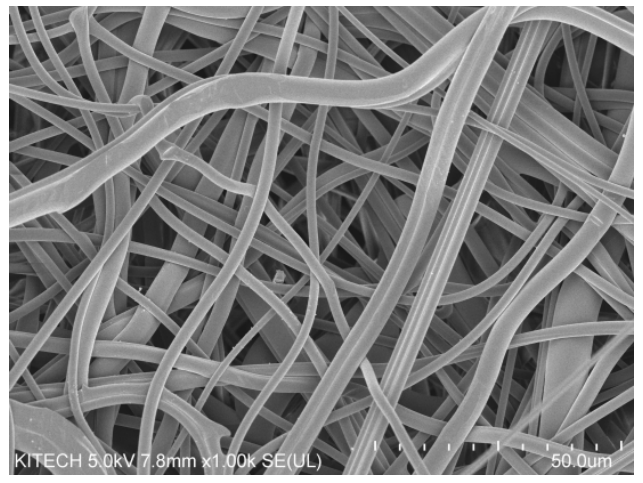

Figure 2. Specifications and SEM images $(\times 1000)$ of melt-blown media by various weight.

\subsubsection{Contact Angle}

The MBNW pre-filter material was analysed using a contact angle measuring instrument. The measurement results of the contact angle of MBNWs are shown in Table 2. The contact angle is the angle formed when the surface comes into contact with the liquid forming MBNW-liquid interface. Based on these measurements, surfaces are classified into hydrophilic, hydrophobic and super-hydrophobic types. Super-hydrophobicity is a phenomenon in which the contact angle of the surface exceeds $150^{\circ}$. Hydrophobicity is a phenomenon in which the contact angle of the surface exceeds $90^{\circ}[20,21]$. The average contact angle of the four MBNW types used in this study was $132.4 \sim 146.6^{\circ}$. All four 
MBNWs made of polypropylene showed hydrophobic properties. As the weight of the MB increased, the contact angle also increased.

Table 2. Contact angle of melt-blown media by various weight.

\begin{tabular}{lcccc}
\hline & $\mathbf{M B}_{1}$ & $\mathbf{M B}_{\mathbf{2}}$ & $\mathbf{M B}_{3}$ & $\mathbf{M B}_{\mathbf{4}}$ \\
\hline $\begin{array}{c}\text { Contact } \\
\text { angle }\left(^{\circ}\right)\end{array}$ & $132.4 \pm 0.3$ & $139.2 \pm 0.5$ & $142.1 \pm 0.4$ & $146.6 \pm 0.2$ \\
& & & & \\
\end{tabular}

\subsubsection{Pore Size}

The MBNW pre-filter materials were analysed using a pore-size analyser. The corresponding curve between flow and pressure was a 'wet curve', and a dry test was conducted to obtain a 'dry curve'. The two curves and semi-dry curves show the pore characteristics of the sample, and the cumulative distribution curve of the pore size can be calculated by accumulating the flow $[22,23]$. The average pore size and pore size distribution of MBs are shown in Table 3. The average pore sizes were between 10.25 and $12.54 \mu \mathrm{m}$, and the bubble pore sizes ranged from $20.03-24.56 \mu \mathrm{m}$. The heavier the weight of the MB, the smaller the mean pore size and bubble pore size. The weight and pore size were inversely proportional.

Table 3. Pore size of melt-blown media by various weight.

\begin{tabular}{ccccc}
\hline Analysis & $\mathbf{M B}_{\mathbf{1}}$ & $\mathbf{M B}_{\mathbf{2}}$ & $\mathbf{M B}_{\mathbf{3}}$ & $\mathbf{M B}_{\mathbf{4}}$ \\
\hline Mean pore size diameter $(\mu \mathrm{m})$ & 12.54 & 11.22 & 10.25 & 10.47 \\
Bubble pore size diameter $(\mu \mathrm{m})$ & 24.56 & 22.08 & 21.06 & 20.03 \\
\hline
\end{tabular}

The pore sizes of the PTFE and MB composites were compared and analysed (Table 4). Only PTFE without MB was measured with an average pore size of $1.08 \mu \mathrm{m}$ and a bubble pore size of $8.13 \mu \mathrm{m}$. It was confirmed that the average pore size when combined with MB was smaller than that when PTFE alone was used inside and outside. Although the pore size of MB alone was larger than that of PTFE, it was confirmed that adding a layer reduces the pore size of the entire filter media. This is the expected result of MB acting as a pre-filtration layer.

Table 4. Pore size of PTFE with melt-blown media by various weights.

\begin{tabular}{cccccc}
\hline Analysis & PTFE & PTFE $+\mathbf{M B}_{\mathbf{1}}$ & $\mathbf{P T F E}+\mathbf{M B}_{\mathbf{2}}$ & $\mathbf{P T F E}+\mathbf{M B}_{\mathbf{3}}$ & $\mathbf{P T F E}+\mathbf{M B}_{\mathbf{4}}$ \\
\hline $\begin{array}{c}\text { Mean pore size } \\
\text { diameter }(\mu \mathrm{m})\end{array}$ & 1.08 & 0.85 & 0.81 & 0.85 & 0.74 \\
$\begin{array}{c}\text { Bubble pore size } \\
\text { diameter }(\mu \mathrm{m})\end{array}$ & 8.13 & 7.49 & 6.12 & 8.45 & 8.18 \\
\hline
\end{tabular}

\subsubsection{Air Permeability}

The air permeability of nonwoven fabrics is an important property that depends on the openness or porosity of the structure. This greatly adjusts the thermal comfort of fabrics. Melt-blown webs have much smaller fibre diameters than spun bond fabrics, which results in smaller pore sizes and lower pore volumes and lower air permeability. However, the air permeability values are still sufficiently high for most applications [13,24]. Generally, higher porosity leads to increased permeability. In this study, as the weight increased, the air permeability decreased, and the pore size influenced the air permeability, even when 
compared with the pore size results. Multiple parameters, such as fibre microstructure, fibre diameter, and nonwoven pore size, affect the air permeability of elastomeric nonwoven composites [13]. Thus, before applying MB to PTFE filter media, the structural characteristics and air permeability of MB were analysed. As shown in Figure 3, the air permeability of $\mathrm{MB}$ was inversely proportional to its weight, and the difference in air permeability between $\mathrm{MB}_{1}$ and $\mathrm{MB}_{4}$ was approximately $30 \mathrm{ccs}$. Figure 4 shows the air permeability of MB when combined with a PTFE membrane. In the result with only MB, the air permeability was significantly reduced below $8 \mathrm{ccs}$, which is determined by the effect of the PTFE membrane, and was 7.6 ccs when only the membrane was measured. By composite with MB on the PTFE membrane, the air permeability was reduced compared to when only PTFE was used. However, this was determined introduce the MB layer, similar to the result of pore size. Additionally, when MB was combined with a PTFE membrane, there was no significant difference in weight compared to MB alone at $6.5 \mathrm{ccs}$ for PTFE with $\mathrm{MB}_{1}$ composite and $5.6 \mathrm{ccs}$ for PTFE with the $\mathrm{MB}_{4}$ composite.

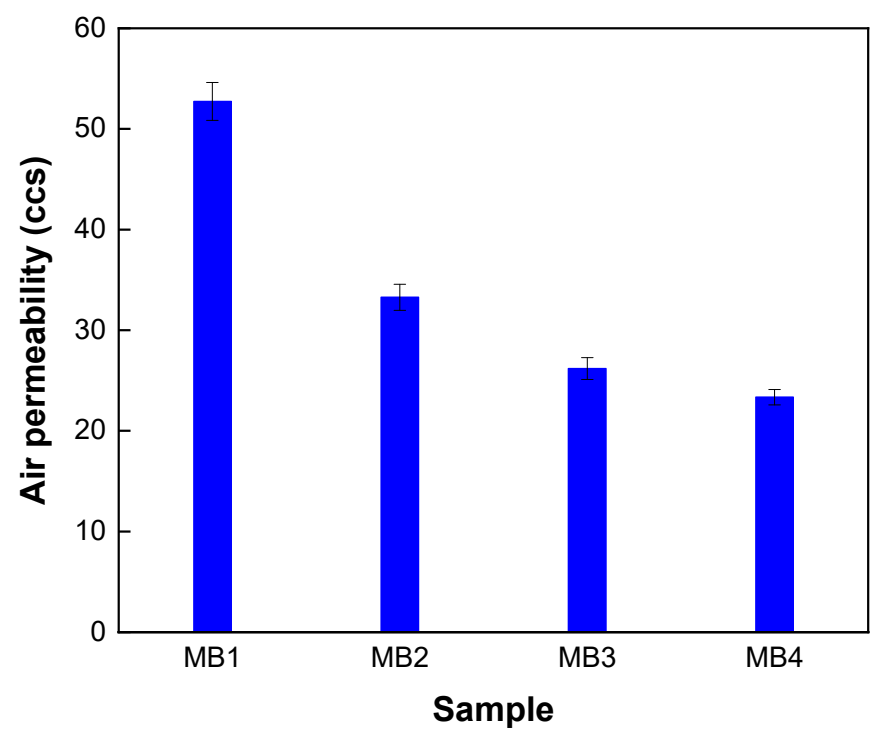

Figure 3. Air permeability of MB by various weights.

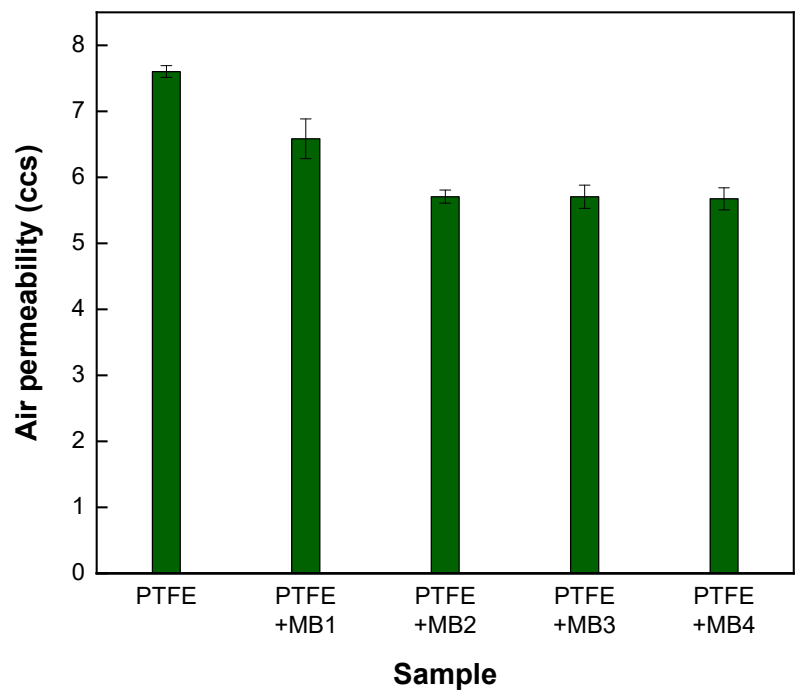

Figure 4. Air permeability of PTFE only and PTFE with MB composites. 


\subsection{Filtration Performance}

Figure 5 shows the performance evaluation measurements of the filtration efficiency by particle sizes from 0.1 to $0.6 \mu \mathrm{m}$. The filter performance was compared and analysed according to $\mathrm{MB}$ weight. The optimal $\mathrm{MB}$ weight should be verified since it is the quality factor for determining the pressure drop and filtration efficiency of the filter [25]. During the evaluation of filtration quality, the pressure drop is particularly important. Conventional PTFE HEPA filters provide high particle removal efficiency. However, filter design must consider such that the pressure drop can increase quickly as accumulated particles clog the filter pores $[23,26,27]$.
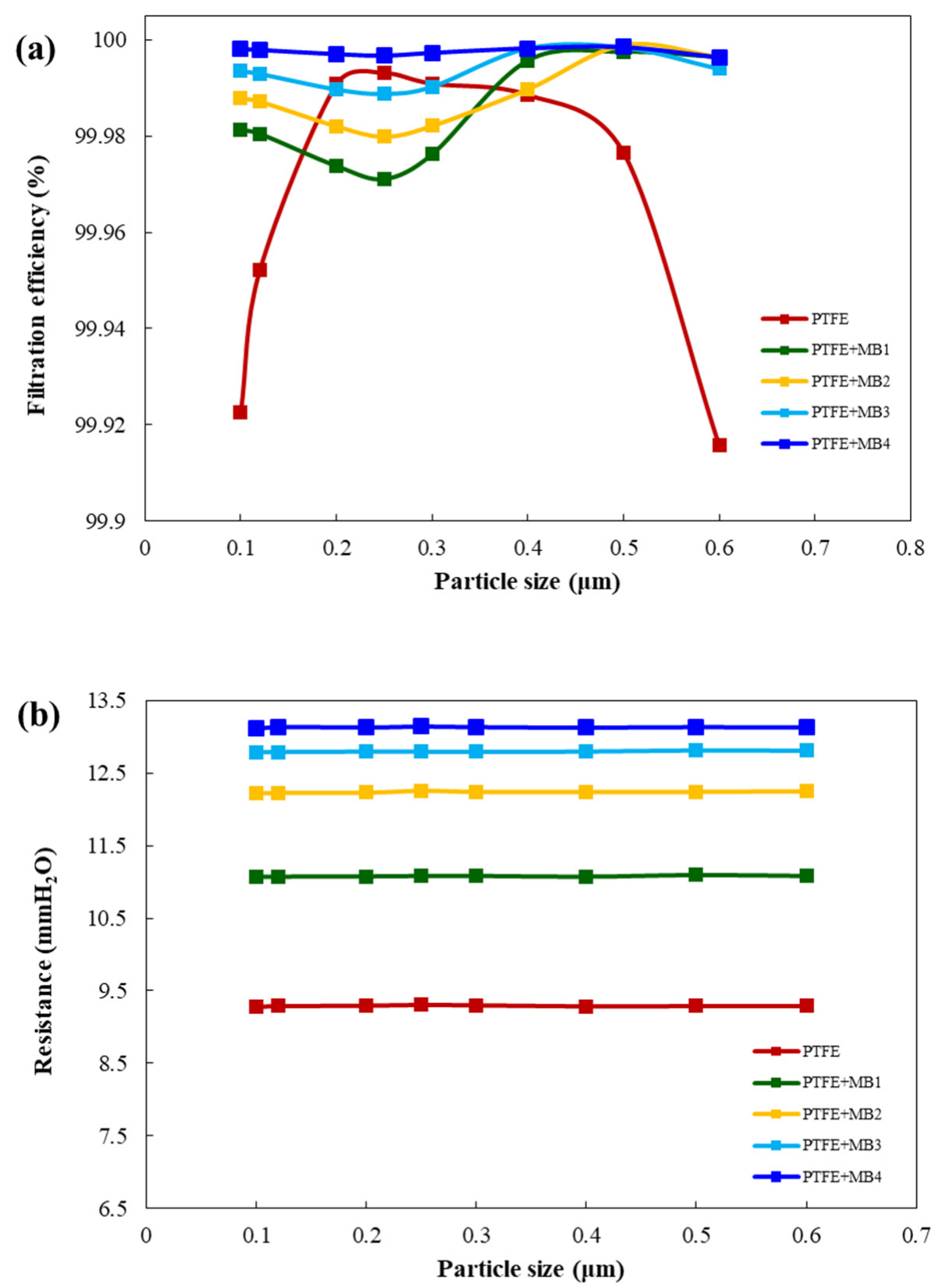

Figure 5. (a) Efficiency and (b) resistance of the PTFE membrane/MB composite on different test dust particle sizes to confirm the MB weight effects according to the EN1822 standards.

The nanoparticle filtration efficiency and resistance of the PTFE membrane and MB composite increased as the MB weight increased. This confirmed that the higher the $\mathrm{MB}$ weight, the smaller the size of the particles. These results suggest that the degree of 
competition in the size of dust molecules also affects the filtration efficiency, and that the filtration efficiency can be increased owing to the electrostatic force of MB [25,28].

\subsection{Dust Loading Test}

Dust loading experiments were conducted at $5.3 \mathrm{~cm} / \mathrm{s}$ face velocity and $2 \mathrm{wt} . \%$ aerosol concentrations. The dust loading test results are important parameters of filter load characteristics related to a higher dust retention capacity life, which means a longer life and both a lower energy consumption and cost of the filter media $[15,29]$.

Figure 6 shows the results of the dust loading performance, and Table 5 shows the amount collected after the loading test. As can be seen in Figure 6 and Table 5, the pressure drop increased modestly when pre-filtration was performed with the MB composite compared to the PTFE membrane only. In addition, when only PTFE was used, the dust collection per area was 1.0. However, when MB was introduced, it increased to a minimum of 3.0 and a maximum of 5.9. A higher dust loading capacity indicates that the prevailing pressure drop occurs more slowly [30,31]. Therefore, among $\mathrm{MB}_{1}-\mathrm{MB}_{4}, \mathrm{MB}_{4}$ had the highest dust retention capacity. With the introduction of $\mathrm{MB}$, the filter is expected to last longer with high efficiency.

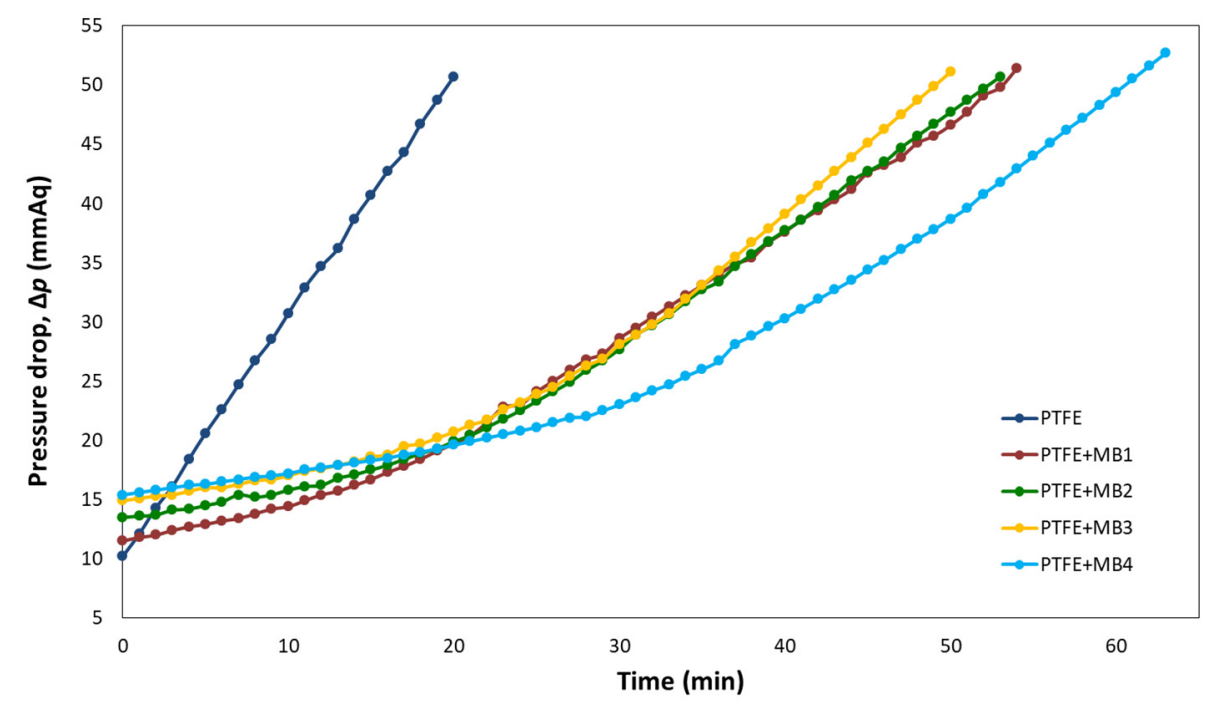

Figure 6. Dust loading performance of PTFE and MB composite at various MB weights.

Table 5. Dust loading capacity.

\begin{tabular}{cccccc}
\hline & PTFE & PTFE $+\mathbf{M B}_{1}$ & PTFE $+\mathbf{M B}_{2}$ & PTFE $+\mathbf{M B}_{3}$ & PTFE $+\mathbf{M B}_{4}$ \\
\hline $\begin{array}{c}\text { Dust loading } \\
\text { capacity }\left(\mathrm{g} / \mathrm{m}^{2}\right)\end{array}$ & 1.0 & 3.0 & 3.8 & 4.4 & 5.9 \\
\hline
\end{tabular}

\subsection{Filter Performance Evaluation in Heating, Ventilation, and Air Conditioning System}

The performance was compared by installing a sample in which $\mathrm{MB}_{4}$ was compounded with PTFE membrane media, the existing glass media, and PTFE media in a commercial HVAC system. As presented in Table 6, the glass media had a slightly higher PTFE media efficiency but had the limitation of applying differential pressure compared to the increased efficiency. The PTFE media developed by improving the shortcomings of glass media, greatly reducing the differential pressure. However, it was not very efficient [3]. It was confirmed in the mounting test that the PTFE media sample in which $\mathrm{MB}$ was compounded compensated for these drawbacks and improved the efficiency to a low differential pressure. In addition, the differential pressure and efficiency were measured after 15, 60, and 120 days when the filter was installed to check the condition of 
the filter. After 120 days, the efficiency of the MB and PTFE composite samples showed efficiencies of $99.984 \%$, which was higher than the glass media which showed an efficiency of $99.978 \%$. Even the MB and PTFE composites showed good filter performance with a differential pressure about two times lower than that of the glass media. It is expected that the performance as a long-life, high-efficiency HEPA filter will be confirmed and that it will be used in an actual industrial environment.

Table 6. Filter performance on contaminated air (outdoor fresh air conditioner: $1680 \mathrm{cmm}$, Air flow: $56 \mathrm{cmm}$ ).

\begin{tabular}{|c|c|c|c|c|c|c|}
\hline & \multicolumn{2}{|c|}{ Glass Media } & \multicolumn{2}{|c|}{ PTFE Media } & \multicolumn{2}{|c|}{ PTFE + MB Composite } \\
\hline & $\begin{array}{c}\text { Pressure } \\
\text { Drop } \\
(\mathrm{mmAq})\end{array}$ & $\begin{array}{c}\text { Efficiency } \\
(\%)\end{array}$ & $\begin{array}{c}\text { Pressure } \\
\text { Drop } \\
(\mathrm{mmAq})\end{array}$ & $\begin{array}{c}\text { Efficiency } \\
(\%)\end{array}$ & $\begin{array}{c}\text { Pressure } \\
\text { Drop } \\
(\mathrm{mmAq})\end{array}$ & $\begin{array}{c}\text { Efficiency } \\
(\%)\end{array}$ \\
\hline $\begin{array}{c}\text { Initial } \\
\text { measurement }\end{array}$ & 28.8 & 99.981 & 14.8 & 99.934 & 14.5 & 99.961 \\
\hline After 15 days & 28.7 & 99.983 & 15.2 & 99.926 & 14.6 & 99.967 \\
\hline After 60 days & 28.3 & 99.993 & 16.5 & 99.966 & 14.6 & 99.986 \\
\hline After 120 days & 28.4 & 99.978 & 17.0 & 99.968 & 14.6 & 99.984 \\
\hline
\end{tabular}

Efficiency for $0.3 \mu \mathrm{m}$ particles.

\section{Conclusions}

In this study, $\mathrm{MB}$ was introduced into the pre-filter to improve the performance of the PTFE membrane based HEPA filter. Four types of MB were produced and analysed according to weight. The comparative analysis of $\mathrm{MB}_{1}, \mathrm{MB}_{2}, \mathrm{MB}_{3}$, and $\mathrm{MB}_{4}$ showed that the filter efficiency was effectively increased when $\mathrm{MB}_{4}$ was introduced into the PTFE membrane. It was confirmed that the DHC performance of PTFE and MB composite samples was increased by approximately three times or more compared to the case where only the PTFE membrane was used, and the life of the filter could be extended. In addition, when evaluating the performance in the actual filter use environment, the differential pressure did not increase even after 120 days, and the efficiency was excellent at $99.984 \%$. As the PTFE membrane-based HEPA filter with MBNW shows excellent performance, thus benefiting the excellent performance and long life of the filter in various HEPA filter usage environments.

Author Contributions: Conceptualization, Y.K.; investigation, E.S. and J.-P.J.; methodology, E.S.; validation, E.S. and Y.K.; formal analysis, J.-P.J.; resources, J.-J.M. and Y.K; data curation, E.S.; visualization, J.-J.M.; supervision, J.-J.M. and Y.K.; project administration, E.S. and J.-P.J.; writingoriginal draft preparation, E.S.; writing — review and editing, Y.K. All authors have read and agreed to the published version of the manuscript.

Funding: This research was funded by the Technology Innovation Program (20010674, Development of ultrahigh performance $(6 \mathrm{~N}) \mathrm{HEPA}$ filter using ePTFE membrane for energy efficiency improvement) funded By the Ministry of Trade, Industry \& Energy (MOTIE, Korea).

Institutional Review Board Statement: Not applicable.

Informed Consent Statement: Not applicable.

Data Availability Statement: Data available in a publicly accessible repository.

Conflicts of Interest: The authors declare no conflict of interest. 


\section{References}

1. Brande, D. Cleanroom certification and particulate testing. In Environmental Monitoring for Cleanrooms and Controlled Environments; CRC Press: Boca Raton, FL, USA, 2016; pp. 65-82.

2. Xu, T.; Lan, C.H.; Jeng, M.S. Performance of large fan-filter units for cleanroom applications. Build. Environ. 2007, 42, 2299-2304. [CrossRef]

3. Zhang, W.; Deng, S.; Wang, Y.; Lin, Z. Dust loading performance of the PTFE HEPA media and its comparison with the glass fibre HEPA media. Aerosol. Air Qual. Res. 2018, 18, 1921-1931. [CrossRef]

4. Zhang, W.; Deng, S.; Wang, Y.; Lin, Z. Modeling the surface filtration pressure drop of PTFE HEPA filter media for low load applications. Build. Environ. 2020, 177, 106905. [CrossRef]

5. Feng, S.; Zhong, Z.; Wang, Y.; Xing, W.; Drioli, E. Progress and perspectives in PTFE membrane: Preparation, modification, and applications. J. Membr. Sci. 2018, 549, 332-349. [CrossRef]

6. Wikol, M.; Hartmann, B.; Brendle, J.; Crane, M.; Beuscher, U.; Brake, J.; Shickel, T.; Gore, W.L. Expanded polytetrafluoroethylene membranes and their applications. Drugs Pharm. Sci. 2008, 174, 619.

7. Galka, N.; Saxena, A. High efficiency air filtration: The growing impact of membranes. Filtr. Sep. 2009, 46, 22-25. [CrossRef]

8. Song, J.; Liu, Z.; Li, Z.; Wu, H. Continuous production and properties of mutil-level nanofiber air filters by blow spinning. RSC Adv. 2020, 10, 19615-19620. [CrossRef]

9. Long, J.; Tang, M.; Sun, Z.; Liang, Y.; Hu, J. Dust loading performance of a novel submicro-fiber composite filter medium for engine. Materials 2018, 11, 2038. [CrossRef]

10. Lee, K.S.; Hasolli, N.; Jeon, S.M.; Lee, J.R.; Kim, K.D.; Park, Y.O.; Hwang, J. Filter layer structure effect on the most penetrating particle size of multilayered flat sheet filter. Powder Technol. 2019, 344, 270-277. [CrossRef]

11. Leung, W.W.F.; Hung, C.H.; Yuen, P.T. Effect of face velocity, nanofiber packing density and thickness on filtration performance of filters with nanofibers coated on a substrate. Sep. Purif. Technol. 2010, 71, 30-37. [CrossRef]

12. Zuo, F.; Tan, D.H.; Wang, Z.; Jeung, S.; Macosko, C.W.; Bates, F.S. Nanofibers from melt blown fiber-in-fiber polymer blends. ACS Macro Lett. 2013, 2, 301-305. [CrossRef]

13. Sikdar, P.; Bhat, G.S.; Hinchliff, D.; Islam, S.; Condon, B. Microstructure and physical properties of composite nonwovens produced by incorporating cotton fibers in elastic spunbond and meltblown webs for medical textiles. J. Ind. Textiles 2021, 15280837211004287. [CrossRef]

14. Chang, D.Q.; Tien, C.Y.; Peng, C.Y.; Tang, M.; Chen, S.C. Development of composite filters with high efficiency, low pressure drop, and high holding capacity PM2.5 filtration. Sep. Purif. Technol. 2019, 212, 699-708. [CrossRef]

15. Tian, X.; Ou, Q.; Pei, C.; Li, Z.; Liu, J.; Liang, Y.; Pui, D.Y. Effect of main-stage filter media selection on the loading performance of a two-stage filtration system. Build. Environ. 2021, 195, 107745. [CrossRef]

16. Chen, C.W.; Huang, S.H.; Chiang, C.M.; Hsiao, T.C.; Chen, C.C. Filter quality of pleated filter cartridges. Ann. Occup. Hyg. 2008, 52, 207-212.

17. Uppal, R.; Bhat, G.; Eash, C.; Akato, K. Meltblown nanofiber media for enhanced quality factor. Fibers Polym. 2013, 14, 660-668. [CrossRef]

18. Todea, A.M.; Schmidt, F.; Schuldt, T.; Asbach, C. Development of a Method to Determine the Fractional Deposition Efficiency of Full-Scale HVAC and HEPA Filter Cassettes for Nanoparticles $\geq 3.5 \mathrm{~nm}$. Atmosphere 2020, 11, 1191. [CrossRef]

19. Yesil, Y.; Bhat, G.S. Structure and mechanical properties of polyethylene melt blown nonwovens. Int. J. Cloth. Sci. Tech. 2016, 28. [CrossRef]

20. Masaeli, E.; Morshed, M.; Tavanai, H. Study of the wettability properties of polypropylene nonwoven mats by low-pressure oxygen plasma treatment. Surf. Interface Anal. 2007, 39, 770-774. [CrossRef]

21. Zhao, T.; Jiang, L. Contact angle measurement of natural materials. Colloids Surfaces B 2018, 161, 324-330. [CrossRef] [PubMed]

22. Zhao, C.; Zhou, X.; Yue, Y. Determination of pore size and pore size distribution on the surface of hollow-fiber filtration membranes: A review of methods. Desalination 2000, 129, 107-123. [CrossRef]

23. Zhou, Z.J.; Zhou, B.; Tseng, C.H.; Hu, S.C.; Shiue, A.; Leggett, G. Evaluation of characterization and filtration performance of air cleaner materials. Int. J. Environ. Sci. Technol. 2020, 18, 1-12. [CrossRef]

24. Gültekin, E.; Çelik, H.I.; Nohut, S.; Elma, S.K. Predicting air permeability and porosity of nonwovens with image processing and artificial intelligence methods. J. Text. Inst. 2020, 111, 1641-1651. [CrossRef]

25. Lim, T.H.; Yeo, S.Y.; Lee, S.H. Multidirectional evaluations of a carbon air filter to verify their lifespan and various performances. J. Aerosol. Sci. 2018, 126, 205-216. [CrossRef]

26. Roh, S.; Park, K.; Kim, J. Design of web-to-web spacing for the reduced pressure drop and effective depth filtration. Polymers 2019, 11, 1822. [CrossRef] [PubMed]

27. Xie, B.; Li, S.; Chu, W.; Liu, C.; Hu, S.; Jin, H.; Zhou, F. Improving filtration and pulse-jet cleaning performance of metal web filter media by coating with polytetrafluoroethylene microporous membrane. Process Saf. Environ. Protect. 2020, 136, 105-114. [CrossRef]

28. Zhang, H.; Liu, J.; Zhang, X.; Huang, C.; Jin, X. Design of electret polypropylene melt blown air filtration material containing nucleating agent for effective PM2. 5 capture. RSC Adv. 2018, 8, 7932-7941. [CrossRef]

29. Sun, Z.; Liang, Y.; He, W.; Jiang, F.; Song, Q.; Tang, M.; Wang, J. Filtration performance and loading capacity of nano-structured composite filter media for applications with high soot concentrations. Sep. Purif. Technol. 2019, 221, 175-182. [CrossRef] 
30. Calle, S.; Contal, P.; Thomas, D.; Bemer, D.; Leclerc, D. Evolutions of efficiency and pressure drop of filter media during clogging and cleaning cycles. Powder Technol. 2002, 128, 213-217. [CrossRef]

31. Yu, J.; Liang, Y.; Tang, M.; Yao, Y. A novel energy-efficient kapok filter paper with high DHC for solid-oil mixed aerosol: Performance and loading behavior evolution mechanism. Sep. Purif. Technol. 2020, 235, 116180. [CrossRef] 